\title{
Architecture distortion score (ADS) in malignancy risk stratification of architecture distortion on contrast-enhanced digital mammography
}

\author{
Yonggeng Goh ${ }^{1}$ (1) $\cdot$ Ching Wan Chan ${ }^{2} \cdot$ Premilla Pillay $^{1} \cdot$ Herng-Sheng Lee $^{3} \cdot$ Huay-Ben Pan $^{4} \cdot$ Bao-Hui Hung ${ }^{4}$. \\ Swee Tian Quek ${ }^{1}$. Chen-Pin $\mathrm{Chou}^{4,5}$
}

Received: 30 June 2020 / Revised: 15 August 2020 / Accepted: 8 October 2020 / Published online: 30 October 2020

(C) The Author(s) 2020

\begin{abstract}
Objective To develop a risk predictor model in evaluation of tomosynthesis-detected architectural distortion (AD) based on characteristics of contrast-enhanced digital mammography (CEDM).

Methods Ninety-four AD lesions on CEDM in combination with tomosynthesis were retrospectively reviewed from 92 consecutive women (mean age, 52.4 years \pm 7.9 ) with abnormal diagnostic or screening mammography. CEDM results were correlated with histology of ADs using cross-tabulation for statistical analysis. Predictors for risk of malignancy from CEDM characteristics (background parenchyma enhancement, degree of AD enhancement, enhancing morphology, size of enhancement, and enhancing spiculations) and patient's age were evaluated using logistic regression. We propose a sum score, termed AD score (ADS), for risk stratification and corresponding suggested BI-RADS category.

Results Thirty-three of ninety-four (35.1\%) of detected AD lesions were malignant. The sensitivity, specificity, PPV, and NPV of CEDM in evaluation of malignant $\mathrm{AD}$ are 100\%, 42.6\%, 48.5\%, and 100\%, respectively. Absence of AD enhancement on CEDM is highly indicative of no underlying malignancy. On multivariate analysis, the predictors on CEDM with statistical significance are (1) marked intensity of AD enhancement (OR, 22.6; 95\%CI 3.1, 166.6; $p=.002)$; and (2) presence of enhancing spiculations (OR, 9.1; 95\%CI 2.2, 36.5; $p=.002)$. A prediction model whose scores (ADS) given by ranking of OR of all predictors with AUC of 0.934 and Brier score of 0.0956 was developed.

Conclusion ADS-based lesion characterization on CEDM enables risk assessment of tomosynthesis-detected AD lesions.

Key Points

- Architecture distortions presenting with marked enhancement intensity and presence of enhancing spiculations are highly associated with risk of malignancy.

- Absence of architecture distortion enhancement in minimal or mild background parenchyma enhancement on CEDM indicates low risk of breast malignancy $(N P V=100 \%)$.
\end{abstract}

Keywords Contrast-enhanced digital mammography · Architecture distortion

Electronic supplementary material The online version of this article (https://doi.org/10.1007/s00330-020-07395-3) contains supplementary material, which is available to authorized users.

Yonggeng Goh

yong_geng_goh@nuhs.edu.sg

$\triangle$ Chen-Pin Chou

chouchenpin@gmail.com

1 Department of Diagnostic Imaging, National University Hospital, Singapore, Singapore

2 Department of Breast Surgery, National University Hospital, Singapore, Singapore
3 Department of Pathology and Laboratory Medicine, Kaohsiung Veterans General Hospital, Kaohsiung, Taiwan

4 Department of Radiology, Kaohsiung Veterans General Hospital, Kaohsiung, Taiwan

5 Department of Medical Laboratory Sciences and Biotechnology, Fooyin University, Kaohsiung, Taiwan 


\section{Abbreviations}

AD Architecture distortion

CEDM Contrast-enhanced digital mammography

DBT Digital breast tomosynthesis

FFDM Full-field digital mammography

ADS Architecture distortion score

\section{Introduction}

Architecture distortion (AD) is the third most common abnormality detected on mammograms $[1,2]$ and is defined as "distortion of the breast parenchymal architecture without a definable mass" according to the Breast Imaging Reporting and Data System (BI-RADS) [3]. There are multiple causes of $\mathrm{AD}$, ranging from breast malignancies to a variety of benign causes such as trauma, post-operative changes, complex sclerosing lesions, or radial scar [4]. Primary AD (defined as cases without history of trauma, intervention, or infection) has been described as a common presentation of non-palpable breast cancer [5]. However, there is conflicting data in the literature regarding its risk of malignancy with positive predictive values (PPVs) ranging from as low as $10 \%$ to as high as $83 \%[1,2,5-7]$. In the absence of a non-invasive modality to accurately differentiate between benign and malignant causes of $\mathrm{AD}$, an invasive procedure such as biopsy or surgical excision is often required. This hence poses a diagnostic and management challenge to breast radiologists and surgeons. In addition, this issue is expected to worsen due to the increased detection of $\mathrm{AD}$ from the growing clinical use of digital breast tomosynthesis (DBT) [6-8].

Therefore, several studies have proposed the use of a contrast-enhanced modality such as magnetic resonance imaging (MRI) to evaluate primary AD [9-11]. This is based on the hypothesis that malignant causes of $\mathrm{AD}$ will demonstrate increased enhancement secondary to angiogenesis, thereby differentiating it from benign causes. In these studies, absence of enhancement of AD correlate on MRI has been shown to be reassuring with high negative predictive values (NPVs) of 80 $100 \%$. However, the high costs and general long waiting times for breast MRI may render it non-feasible for many healthcare settings and institutions in evaluation of all $\mathrm{AD}$ cases.

Contrast-enhanced digital mammography (CEDM) is a new and emerging breast imaging modality which uses contrast media and the principle of dual-energy subtraction to evaluate enhancement of breast lesions. CEDM is less expensive compared to MRI and has demonstrated results comparable to MRI in many settings of breast imaging such as lesion characterization, local staging, and evaluating response to neoadjuvant therapy [12-16]. CEDM, as a cost-effective substitute, could evaluate $\mathrm{AD}$ in a way similar to MRI and provides a perfect correlation of mammographic and contrastenhanced findings. However, there is a lack of literature to support its use. As far as we know, there is only a single study published to date and the sample size was small [17].

Hence, this study, with a larger sample size is designed to investigate the diagnostic capabilities of CEDM in the evaluation of $\mathrm{AD}$ detected on $\mathrm{DBT}$, in the hope to potentially reduce the number and need for biopsy or surgical excision in patients with benign causes of AD. In this study, we also aim to develop a practical scoring model to stratify the malignant risk of $\mathrm{AD}$ on $\mathrm{CEDM}$ based on each imaging characteristic.

\section{Materials and methods}

This retrospective Health Insurance Portability and Accountability Act-compliant review was approved by our institutional review board. The need for informed consent was waived.

\section{Patient selection}

Retrospective study of radiology database identified 700 consecutive CEDM examinations in combination with tomosynthesis for abnormal diagnostic or screening imaging findings at the Department of Radiology, Kaohsiung Veterans General Hospital, from February 2012 to Nov 2019. The important findings (mass, calcifications, focal asymmetry, AD) on FFDM (full-field digital mammography) and DBT were based on independent review of three board-certified breast radiologists (C.C.P., H.B.P., and B.H.H.) trained on breast imaging with 17,27 , and 5 years of experience. AD detected on FFDM and DBT was achieved when at least two out of three radiologists had the same imaging report of $\mathrm{AD}$. Lesions seen at both FFDM and DBT were considered to be FFDM detected. Results of CEDM for all detected ADs were correlated with histopathological findings from biopsy or surgical excision results. Patients who did not undergo biopsy or surgery due to technique limitations or other reasons would require a minimum imaging follow-up (DBT and breast MRI) of 2 years to ascertain true benign status. The exclusion criteria were (1) patients lacking biopsy proof and 2 years of DBT follow-up; (2) ADs secondary to previous surgery.

\section{CEDM image acquisition protocol}

All CEDM images were acquired using a mammography system (Selenia Dimensions, Hologic) with dual-energy exposure. The contrast medium Omnipaque 350 (GE Healthcare Inc.) was injected into patients via an automatic power injector (Vistron CT injection system, Medrad) at a volume of $1.5 \mathrm{ml} /$ $\mathrm{kg}$ of body weight and rate of $3 \mathrm{ml} / \mathrm{s}$ through a peripheral intravenous cannula. After completion, patients were disconnected from the automatic power injector. 
The CEDM images were obtained starting at 2 min after contrast medium injection. Mediolateral oblique (MLO) and craniocaudal (CC) views of the breast with the lesion of concern would be obtained first followed by CC and MLO views of the contralateral breast. Two exposures (i.e., high-energy at 45-49 kVp and low-energy beam at 26-32 kVp) were obtained almost simultaneously for each view and a subtracted image between the two was generated to visualize contrast enhancement of both breasts. The image acquisition of all 4 views was completed within $7 \mathrm{~min}$.

\section{CEDM image interpretation and analysis}

The subtracted CEDM images were reviewed by 3 breast radiologists (C.C.P., H.B.P., and B.H.H.) who were blinded to final histology results. Consensus expert opinion was taken to be the agreement of at least 2 of 3 of the participating radiologists. Firstly, the background parenchymal enhancement (BPE) of both breasts was evaluated and categorized as follows: (1) minimal; (2) mild; (3) moderate; or (4) marked (Fig. 1).

Secondly, the enhancement characteristics of AD were evaluated. These are based on characteristics such as (1) enhancement intensity; (2) size; (3) morphology; and (4) margins. For intensity of contrast enhancement, the lesions were graded as follows: (1) absent; (2) mild; (3) moderate; or (4) marked using our standard reference image for CEDM enhancement (Fig. 2). The sizes of the $\mathrm{AD}$ enhancement based on only CEDM images were measured using a three-monitor workstation (SecurViewDx, Hologic) capable of displaying full-field digital mammography (FFDM), DBT, and CEDM. Characteristics of non-contrast mammographic images were not used for data analysis.

\section{Minimal}

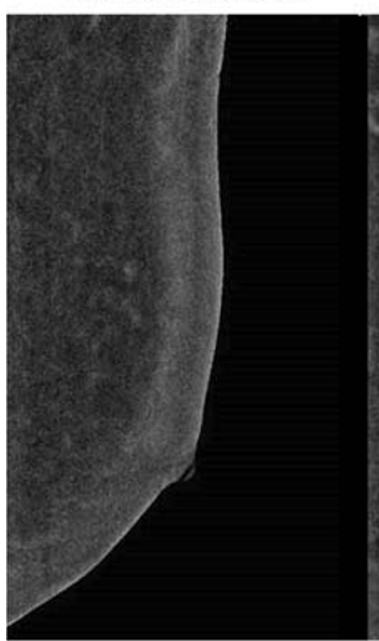

Mild

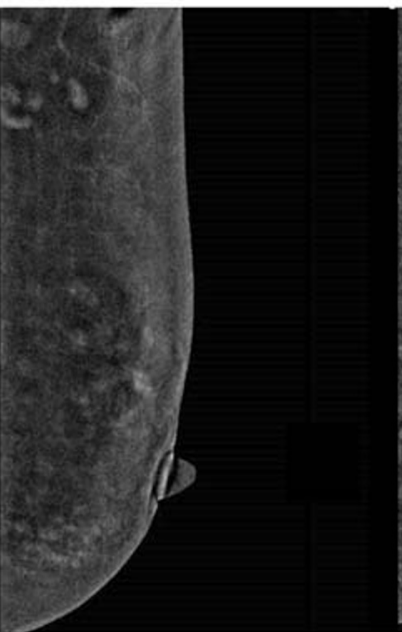

The morphology of enhancement was categorized as follows: (1) focus (<5 mm); (2) mass; or (3) non-mass enhancement according to lexicons as described for breast MRI developed by the American College of Radiology [3] (Fig. 3). Lastly, the AD lesions which demonstrated mass or nonmass enhancement were assessed for the presence or absence of enhancing spiculations (Fig. 3).

\section{Data and statistical analysis}

The presence or absence of AD enhancement on CEDM was compared to histological findings obtained from available biopsy or surgical excision reports in a categorical format (i.e., benign or malignant). In cases where no histological correlation was observed, patients were followed up for the next 2 years to confirm its true benign status. Cross-tabulation was performed to evaluate the overall diagnostic accuracy, sensitivity, specificity, PPV, and NPV of CEDM in evaluation of AD. In this study, surgical results of high-risk lesions such as atypical ductal hyperplasia $(\mathrm{ADH})$ and lobular carcinoma in situ (LCIS) were classified as benign cases.

Predictors for AD from CEDM characteristics (i.e., BPE, intensity of contrast enhancement, enhancing morphology, size of enhancement, and enhancing spiculations), patient's age, and risks of malignancy were also evaluated using univariate and multivariate logistic regression analyses. Odds ratios (OR) with $95 \%$ confidence intervals (CI) were presented. Receiver operating characteristic curve (ROC) analysis and Youden index were performed for size of AD lesion enhancement on CEDM to determine the optimal cutoff value for maximizing specificity and sensitivity. Prediction models for AD were developed. A sum score, termed AD score (ADS), was derived from the prediction model for risk stratification.

Fig. 1 Representative contrast-enhanced digital mammography (CEDM) show breasts with minimal, mild, moderate, and marked background parenchyma enhancement (BPE) 


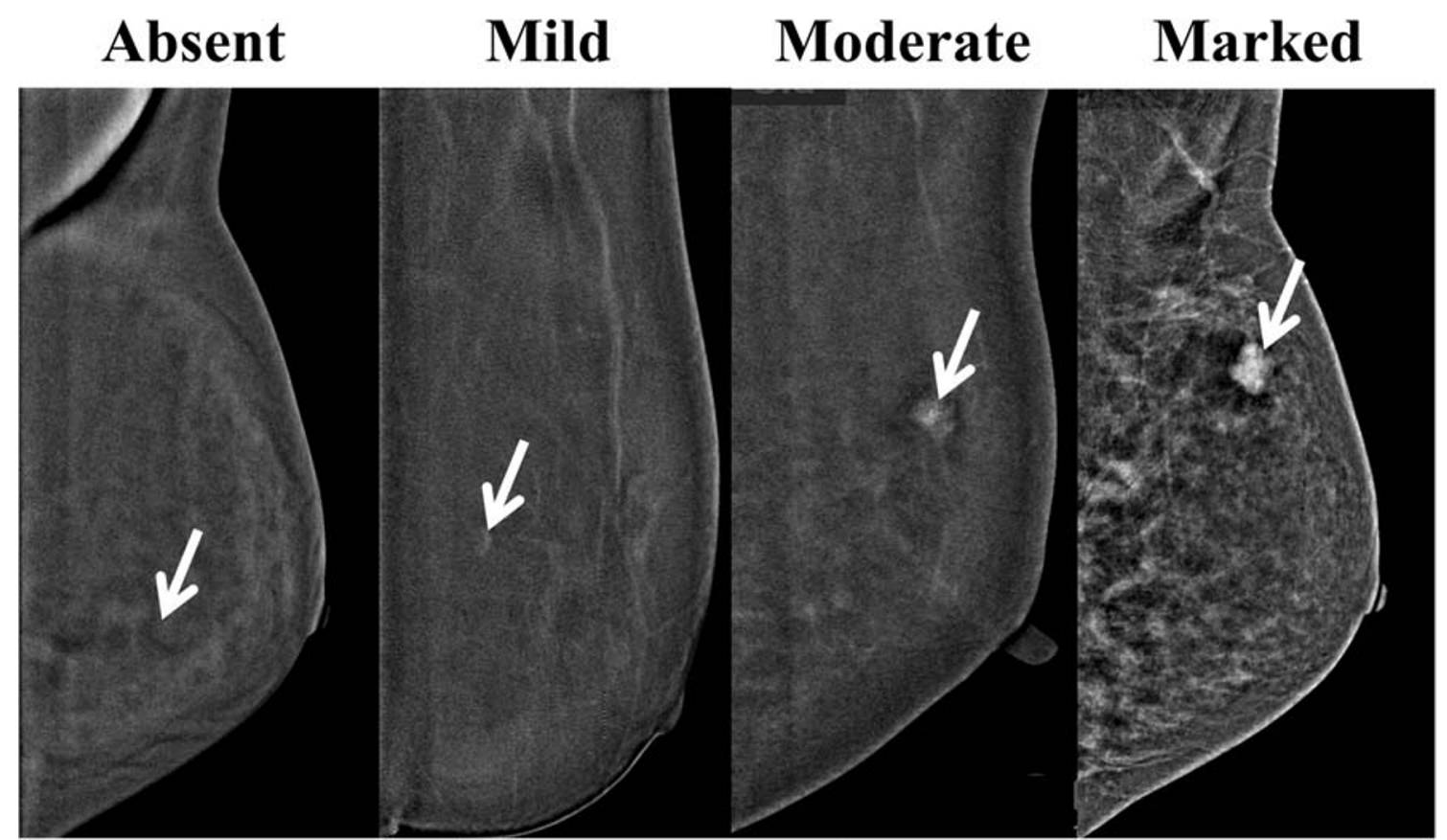

Fig. 2 A standard reference image for degree of common lesion enhancement on contrast-enhanced digital mammography (CEDM) with absent, mild, moderate, and marked

All the statistical analyses were performed by using the SPSS software (Version 22; PASW Statistics) with statistical significance set at $p<0.05$.

\section{Results}

A total of 851 target lesions in 700 women were noted in our CEDM database. After excluding 753 mammographic lesions (mass, calcifications, focal asymmetry, and negative findings) other than $\mathrm{AD}$ and 4 lesions with incomplete imaging exams, there were a total of $94 \mathrm{AD}$ lesions (61 benign, 33 malignant) detected from 92 women (1 patient had 3 lesions). Mean age of the enrolled patients was $52.4 \pm 7.9$ (SD) years. The 33 breast malignancies were confirmed by histopathological diagnosis and followed by surgical management. Fifty-one (83.6\%) of 61 benign lesions had histopathological correlation with imaging-guided biopsy (ultrasound, tomosynthesis, MRI) or surgical excision after wire localization. The remaining $10(16.4 \%)$ lesions with no histological correlation were followed up closely with DBT for at least 2 years to ascertain true benign status. There were no patients excluded from this study due to previous surgery or due to the lack of follow-up/surgery. Twenty-one $(22.3 \%)$ of $94 \mathrm{AD}$ lesions were detected on DBT only. Patient characteristics and histopathology of $\mathrm{AD}$ lesions included in the analysis are shown in Table 1.

\section{Benign lesions}

Fibrocystic change with stromal fibrosis $(23 / 61,37.7 \%)$ forms the majority of benign lesions in our study. Of the 17 radial scars, 6 were diagnosed via direct surgical excision after wire localization with no evidence of disease upgrade after surgery. The remaining 11 radial scar lesions were diagnosed via a combination of DBT-guided VABB (vacuum-assisted breast biopsy) and negative DBT results on follow-up (mean, 30 months; range, 11-85 months). Ten lesions with no histological diagnoses were diagnosed as benign on additional breast MRI or on follow-up imaging. In these 10 patients, 6 underwent breast MRI for very subtle lesions on both DBT and CEDM. The final assessments of these 6 lesions were regarded as BI-RADS category $1-2$ on the basis of MRI findings. The remaining 4 of 10 patients had small AD lesions with no suspicious enhancement on CEDM. These were highly indicative of no underlying malignancy and would have been difficult for tomosynthesis-guided biopsy. Nonetheless, all 10 lesions were followed up for at least 2 consecutive years with DBT and ultrasound to affirm the absence of malignancy.

\section{High-risk lesions}

High-risk lesions (5/61) (i.e., atypical ductal hyperplasia ( $n=$ 2), lobular carcinoma in situ ( $n=2)$, flat epithelial atypia (FEA) $(n=1))$ were considered benign in our study and made up the minority $(8.2 \%)$ of benign lesions. All 5 lesions were diagnosed via $\mathrm{VABB}$ and subsequent surgical excisions. High-risk lesions would be re-classified as malignant if lesions exhibit 


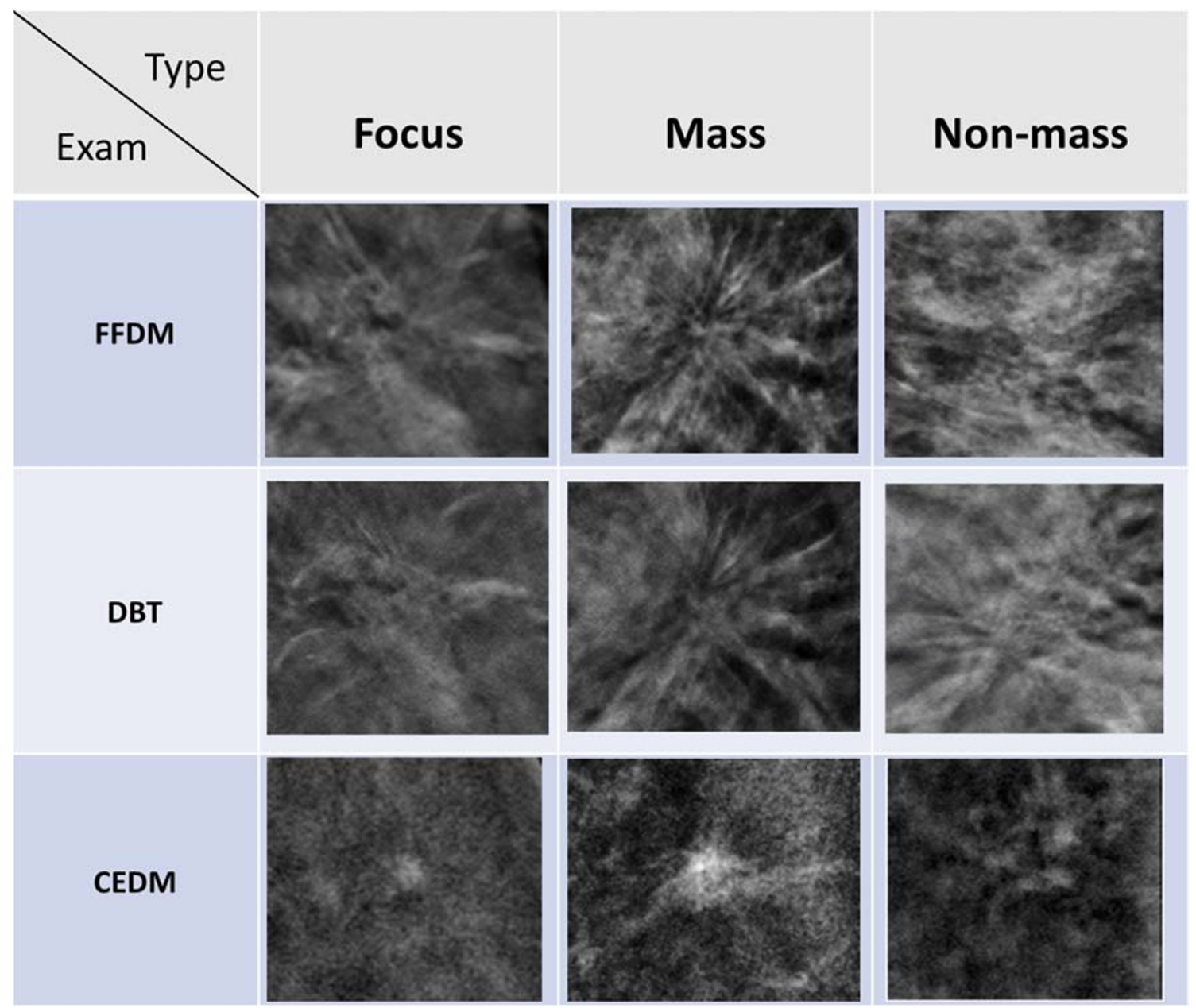

Fig. 3 Images of AD lesions at full-field digital mammography (FFDM), digital breast tomosynthesis (DBT), and contrast-enhanced digital mammography (CEDM). AD enhancing pattens at CEDM are classified into

pleomorphism or evidence of disease upgrade upon excision. However, none of the 5 high-risk lesions showed disease upgrade on excision and was classified as benign in this study.

\section{CEDM characteristics}

The following CEDM characteristics were collected as variables during data analysis, namely (1) CEDM BPE; (2) size of $\mathrm{AD}$ on CEDM; (3) CEDM enhancement morphology; (4) AD enhancement intensity; and (5) presence/absence of enhancing spiculation on CEDM. Cross-tabulation was performed for each of these variables to determine the sensitivity, specificity, PPV, and NPV of CEDM in evaluation of AD. In this study, the variables which demonstrate the highest PPV for malignancy on CEDM are as follows: (1) marked enhancement intensity of AD lesion on CEDM (PPV $=89.5 \%$ (17/ 19)); (2) presence of enhancing spiculations ( $\mathrm{PPV}=70.6 \%$ (24/34)); (3) mass/non-mass enhancing morphology on CEDM (PPV $=55.6 \%(30 / 54))$. These results are summarized in Table 2. three groups: focus $(<5 \mathrm{~mm})$, mass with and without enhancing spiculations, and non-mass

\section{Statistical analysis and prediction model}

Overall, the sensitivity, specificity, PPV, and NPV of CEDM in the evaluation of $\mathrm{AD}$ are $100 \%$ (95\% CI $89.4 \%, 100 \%)$, $42.6 \%$ (95\% CI $30.0 \%, 55.9 \%$ ), $48.5 \%$ (95\% CI $36.2 \%$, $61.0 \%$ ), and $100 \%$ (95\%CI $86.8 \%, 100 \%)$, respectively (Table 2). A ROC curve analysis (Fig. 4) for AD size on CEDM was performed which demonstrated an optimal cutoff size for enhancing $\mathrm{AD}$ lesion on CEDM to be approximately $0.70 \mathrm{~cm}$ (sensitivity, $88.6 \%$; specificity, $67.8 \%$ ).

At univariate analysis, the following features on CEDM were shown to demonstrate a significant association with breast malignancy: (1) size of contrast enhancement $(\geq 0.7 \mathrm{~cm})$; (2) enhancing morphology (mass/non-mass enhancement); (3) enhancement intensity (moderate to marked); and (4) presence of enhancing spiculations. However, multivariate analysis showed that breast malignancy was significantly associated with (1) marked intensity of AD lesion on CEDM (OR 22.6; $95 \%$ CI $3.1,166.6 ; p=.002)$; and (2) presence of 
Table 1 Patient characteristics and histopathology results of 94 AD lesions

\begin{tabular}{lc}
\hline Parameter & Value \\
\hline Age (years) & \\
Mean \pm SD & $52.4 \pm 7.9$ \\
Range & $30-69$ \\
Breast density category & \\
Almost entirely fatty & $2(2 \%)$ \\
Scattered densities & $2(2 \%)$ \\
Heterogeneous dense & $79(84 \%)$ \\
Extremely dense & $11(12 \%)$ \\
Size of enhancing AD (cm) & \\
Mean \pm SD & $1.06 \pm 1.13$ \\
Range & $0.1-6.2$ \\
Malignancy $(n=33)$ & \\
Invasive ductal carcinoma & $18(54.5 \%)$ \\
Ductal carcinoma in situ & $10(30.3 \%)$ \\
Invasive lobular carcinoma & $4(12.1 \%)$ \\
Tubular carcinoma & $1(3.0 \%)$ \\
Benign disease $(n=61)$ & \\
Fibrocystic change with stromal fibrosis & $23(37.7 \%)$ \\
Radial scar & $17(27.9 \%)$ \\
Benign by imaging follow-up $\geq 2$ years & $10(16.4 \%)$ \\
Sclerosing adenosis & $6(9.8 \%)$ \\
High-risk lesions (ADH or LCIS) & $5(8.2 \%)$ \\
\hline A architection & \\
\hline
\end{tabular}

$A D$ architectural distortion, $S D$ standard deviation, $A D H$ atypical ductal hyperplasia, LCIS lobular carcinoma in situ

enhancing spiculations (OR 9.1; 95\% CI 2.2, 36.5; $p=.002)$. Findings are summarized in Table 3.

With all these findings, a prediction model with all original variables in logistic regression (herein referred to as full model) was created using area under the ROC curve (AUC) as benchmark (AUC of 0.934 and a Brier score of 0.0956). Next, after categorizing all variables, a prediction model based on the ranking of the OR with an AUC of 0.919 and a Brier score of 0.0971 was created. A sum score, termed AD score (ADS), was derived from the prediction model for risk stratification in view of its clinical utility in assisting prediction of malignant risk (Table 4). There is no significant differences between the 2 prediction model scores (see Fig. 4). To facilitate better understanding of the prediction model, pictorial case examples with clinical application of AD scores are demonstrated in Fig. 5 and Figs. 6 and 7 (see supplementary data).

\section{Discussion}

Many imaging modalities have been studied in the evaluation of $\mathrm{AD}$, including $\mathrm{DBT}$ with or without ultrasound correlates $[6,7,18-20]$ and MRI $[11,21]$, but each has its own limitation. With the increased detection of $\mathrm{AD}$ from growing use of DBT, there is an unmet clinical need for a cost-effective imaging modality for characterization of $\mathrm{AD}$ lesions to reduce the need for unnecessary invasive procedures such as biopsy or surgical excision. Our results demonstrated that CEDM can be a very useful adjunct tool in assessment of AD lesions. We found that $\mathrm{AD}$ lesion with no or mild contrast enhancement on CEDM has a lower chance of breast malignancy than that with moderate or marked contrast enhancement. The use of an accurate prediction model $(\mathrm{AUC}=0.921$, Brier score $=$ 0.099) can assist in decision making during assessment of $\mathrm{AD}$ lesions according to BI-RADS categories for breast radiologists or surgeons (see Figs. 6 and 7 under Supplementary Data).

Several studies have demonstrated DBT-only AD to have a lower PPV for malignancy as compared to mammographydetected AD $[5,6]$. This could partially explain the relatively low PPV of $48.5 \%$ on CEDM in our study as $22.3 \%$ of $\mathrm{AD}$ lesions were detected on DBT only. The relatively low PPV for $\mathrm{AD}$ lesions on $\mathrm{CEDM}$ also suggests that not all $\mathrm{AD}$ lesions which enhance on CEDM would warrant a biopsy or surgical excision to achieve histological diagnosis. The associations between characteristics of $\mathrm{AD}$ on CEDM and risk of malignancy were assessed using a multivariate analysis. This demonstrated 2 factors on CEDM which showed statistical significance: (1) marked enhancement intensity of AD on CEDM (rank score $=5$ ); and (2) presence of enhancing spiculations on CEDM (rank score $=4$ ).

Marked enhancement intensity of AD on CEDM has demonstrated a significant association with malignancy in our study. While many benign breast lesions enhance (e.g., mild hyperplasia or proliferative lesions without atypia), these usually only demonstrate mild enhancement on contrastenhanced modalities such as MRI [22] as opposed to marked enhancement of malignant lesions due to underlying angiogenesis. In our study, marked enhancement on CEDM was a significant factor with an OR of 22.6 (95\%CI 3.1, 166.6; $p=.002$ ). Of note, the assessment of enhancement intensity in our study remains subjective and further studies with use of quantitative measures or software may help to better stratify malignant risk in the future [23].

Secondly, spiculated margins of radiologically detected masses have been well-known morphologic criteria for breast malignancy $[22,24]$. While this is largely unsurprising for masses, enhancing spiculations for AD have not been well described on CEDM. In our study, we investigated the association of enhancing spiculations on CEDM with risk of malignancy which demonstrated an OR of 9.1 (95\% CI 2.2, 36.5; $p=.002$ ).

The presence of moderate to marked BPE has been well known to cause considerable effect in detection of breast malignancy on contrast-enhanced modalities such as MRI [25, 26]. Moderate to marked BPE could potentially mask 
Table 2 Cross-tabulation table of CEDM characteristics with histopathology $(N=94)$

\begin{tabular}{|c|c|c|c|c|}
\hline & & $\begin{array}{l}\text { Histology } \\
\text { Benign }\end{array}$ & Malignant & $\operatorname{PPV}(\%)$ \\
\hline \multirow[t]{2}{*}{ Background parenchyma enhancement (BPE) } & Minimal/mild & 53 & 26 & $32.9(26 / 79)$ \\
\hline & Moderate/marked & 8 & 7 & $46.7(7 / 15)$ \\
\hline \multirow[t]{2}{*}{ Size of AD enhancement } & $<0.7 \mathrm{CM}$ & 37 & 4 & $9.8(4 / 41)$ \\
\hline & $\geq 0.7 \mathrm{CM}$ & 24 & 29 & $54.7(29 / 53)$ \\
\hline \multirow[t]{3}{*}{ Morphology } & Absent & 26 & 0 & 0 \\
\hline & Focus & 11 & 3 & $21.4(3 / 14)$ \\
\hline & Mass/non-mass & 24 & 30 & $55.6(30 / 54)$ \\
\hline \multirow[t]{3}{*}{ AD enhancement intensity } & Absent/mild & 47 & 7 & $13.0(7 / 54)$ \\
\hline & Moderate & 12 & 9 & $42.9(9 / 21)$ \\
\hline & Marked & 2 & 17 & $89.5(17 / 19)$ \\
\hline \multirow[t]{2}{*}{ Enhancing spiculations } & Present & 10 & 24 & $70.6(24 / 34)$ \\
\hline & Absent & 51 & 9 & $15.0(9 / 60)$ \\
\hline \multirow[t]{4}{*}{ CEDM enhancement } & Absent & 26 & 0 & 0 \\
\hline & Present & 35 & 33 & $48.5(33 / 68)$ \\
\hline & \multicolumn{3}{|c|}{ Overall AD evaluation with CEDM $(N=94)$} & \\
\hline & Result & 95\% CI (lower) & 95\% CI (upper) & \\
\hline Sensitivity & $100 \%(33 / 33)$ & $89.4 \%$ & $100 \%$ & \\
\hline Specificity & $42.6 \%(26 / 61)$ & $30.0 \%$ & $55.9 \%$ & \\
\hline PPV & $48.5 \%(33 / 68)$ & $36.2 \%$ & $61.0 \%$ & \\
\hline NPV & $100 \%(26 / 26)$ & $86.8 \%$ & $100 \%$ & \\
\hline Accuracy & $62.8 \%(59 / 94)$ & $52.2 \%$ & $72.5 \%$ & \\
\hline
\end{tabular}

underlying malignancies, resulting in difficult evaluation of target lesions. While our study did not demonstrate any significant association of malignancy, moderate to marked BPE $($ rank score $=3$ ) remains a significant clinical factor and a potential pitfall while evaluating contrast enhancement of lesion on CEDM images.

Contrary to the relative low PPV, our results demonstrated an extremely high NPV of $100 \%$ in $26(27.7 \%)$ of 94 lesions which showed no suspicious enhancement on CEDM. Bearing in mind that the moderate to marked BPE could mask underlying malignancies, the results suggest that the absence of AD lesion enhancement on CEDM on a background of minimal or mild BPE has a lower risk of breast malignancy. However, invasive procedures should be considered for any AD lesion with clinical symptoms or other imaging abnormalities despite a low AD score on CEDM.

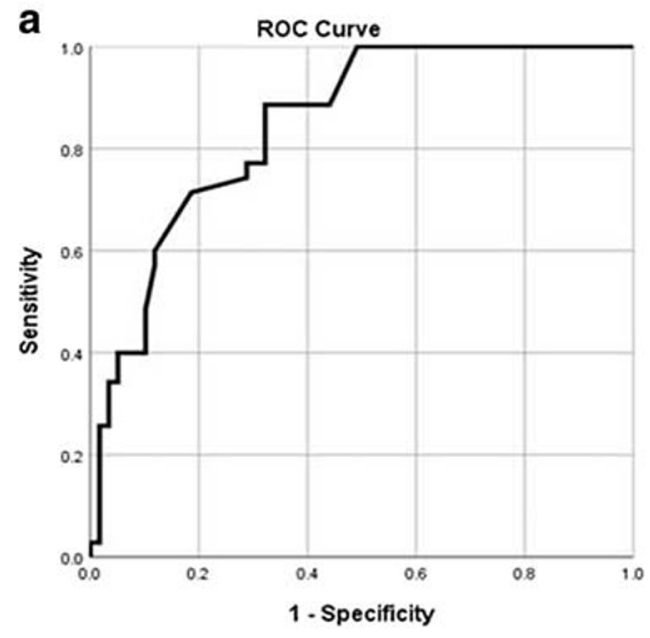

Fig. 4 (a) ROC curve for enhancing size of AD on CEDM demonstrates an optimal cutoff value of approximately $0.7 \mathrm{~cm}$ (sensitivity $88.6 \%$; specificity, 67.8\%). (b) ROC curves for both full model and prediction

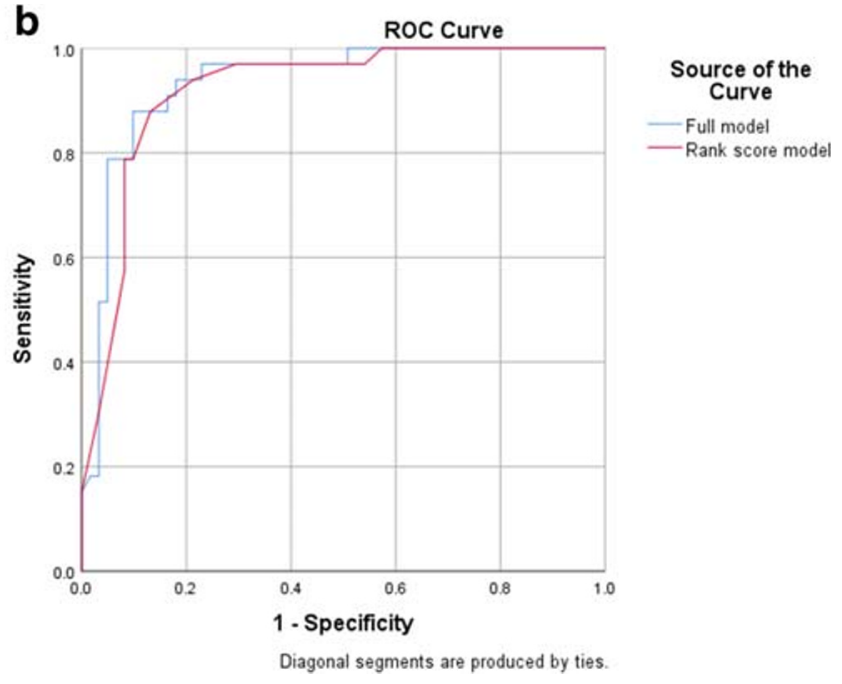

model based on the ranking of the odds ratios (i.e., ADS (architecture distortion score) on CEDM show no statistical difference between the two) 
Table 3 Association between breast malignancy and AD characteristic on CEDM

\begin{tabular}{|c|c|c|c|c|c|c|}
\hline \multirow[b]{2}{*}{ Characteristic } & \multicolumn{2}{|c|}{ Histopathology } & \multicolumn{2}{|l|}{ Unadjusted analysis } & \multicolumn{2}{|l|}{ Adjusted analysis } \\
\hline & Benign & Malignant & Odds ratio $(95 \% \mathrm{CI})$ & $p$ value & Odds ratio $(95 \% \mathrm{CI})$ & $p$ value \\
\hline Mean age (SD) & $52.1(7.1)$ & $52.8(9.3)$ & $1.01(0.96-1.07)$ & 0.692 & $1.05(0.96-1.14)$ & 0.279 \\
\hline \multicolumn{7}{|l|}{$\begin{array}{l}\text { Background parenchyma } \\
\text { enhancement (BPE) }\end{array}$} \\
\hline - Minimal + mild & $53(67.1 \%)$ & $26(32.9 \%)$ & 1.0 & $\ldots$ & 1.0 & $\ldots$ \\
\hline - Moderate + marked & $8(53.3 \%)$ & $7(46.7 \%)$ & $1.8(0.6-5.5)$ & 0.310 & $2.4(0.4-13.9)$ & 0.315 \\
\hline \multicolumn{7}{|l|}{ Size of AD enhancement } \\
\hline$-<0.7 \mathrm{~cm}$ & $37(90.2 \%)$ & $4(9.8 \%)$ & 1.0 & $\ldots$ & 1.0 & $\ldots$ \\
\hline$-\geq 0.7 \mathrm{~cm}$ & $24(45.3 \%)$ & $29(54.7 \%)$ & $11.2(3.5-35.8)$ & $<0.001 * * *$ & $1.5(0.1-15.5)$ & 0.738 \\
\hline \multicolumn{7}{|l|}{ Enhancing morphology } \\
\hline - Absent & $26(100 \%)$ & $0(0 \%)$ & NA & NA & NA & NA \\
\hline - Focus & $11(78.6 \%)$ & $3(21.4 \%)$ & 1.0 & $\ldots$ & 1.0 & $\ldots$ \\
\hline - Mass/non-mass & $24(44.4 \%)$ & $30(55.6 \%)$ & $4.6(1.1-18.3)$ & $0.031 *$ & $1.8(0.2-18.5)$ & 0.638 \\
\hline \multicolumn{7}{|c|}{ Enhancement intensity of AD } \\
\hline - Absent + mild & $47(87.0 \%)$ & $7(13.0 \%)$ & 1.0 & $\ldots$ & 1.0 & $\ldots$ \\
\hline - Moderate & $12(57.1 \%)$ & $9(42.9 \%)$ & $5.0(1.6-16.3)$ & $0.007 * *$ & $1.4(0.3-6.5)$ & 0.696 \\
\hline - Marked & $2(10.5 \%)$ & $17(89.5 \%)$ & $57.1(10.8-302.1)$ & $<0.001 * * *$ & $22.6(3.1-166.6)$ & $0.002 * *$ \\
\hline \multicolumn{7}{|c|}{ Enhancing spiculations of $\mathrm{AD}$} \\
\hline - Present & $10(29.4 \%)$ & $24(70.6 \%)$ & $13.6(4.9-37.8)$ & $<0.001 * * *$ & $9.1(2.2-36.5)$ & $0.002 * *$ \\
\hline - Absent & $51(85.0 \%)$ & $9(15.0 \%)$ & 1.0 & $\ldots$ & 1.0 & $\ldots$ \\
\hline
\end{tabular}

$A D$ architectural distortion, $C E D M$ contrast-enhanced digital mammography, $C I$ confidence interval, $N A$ not available, $S D$ standard deviation

$* p<0.05, * * p<0.01, * * * p<0.001$

Table 4 Architectural distortion score (ADS) and corresponding suggested BI-RADS category in predicting breast malignancy for AD lesions detected at digital breast tomosynthesis

\begin{tabular}{|c|c|c|c|}
\hline Characteristic & Odds ratio & Weight & $\begin{array}{l}\text { Rank/ } \\
\text { score }\end{array}$ \\
\hline \multicolumn{4}{|c|}{$\begin{array}{l}\text { CEDM background } \\
\text { parenchyma enhancement }\end{array}$} \\
\hline - Minimal & 1.0 & 0 & 0 \\
\hline - Mild & 1.0 & 1 & 1 \\
\hline - Moderate or marked & 2.9 & 3 & 3 \\
\hline \multicolumn{4}{|l|}{ Size of AD on CEDM } \\
\hline$-<0.7 \mathrm{~cm}$ & 1 & 0 & 0 \\
\hline$-\geq 0.7 \mathrm{~cm}$ & 1.8 & 2 & 2 \\
\hline \multicolumn{4}{|l|}{ Enhancing morphology\# } \\
\hline - Focus & NA & NA & 6 \\
\hline - Mass/non-mass & NA & NA & 7 \\
\hline \multicolumn{4}{|l|}{ Enhancement intensity } \\
\hline - Absent & NA & 0 & 0 \\
\hline - Mild & NA & 1 & 1 \\
\hline - Moderate & 1.7 & 2 & 2 \\
\hline - Marked & 30.2 & 30 & 5 \\
\hline \multicolumn{4}{|l|}{$\begin{array}{l}\text { Enhancing spiculations of } \\
\text { AD on CEDM }\end{array}$} \\
\hline - Present & 13.9 & 14 & 4 \\
\hline - Absent & 1.0 & 0 & 0 \\
\hline Age $(>52$ years $)$ & 3.1 & 3 & 3 \\
\hline
\end{tabular}

$A D$ architectural distortion, $C E D M$ contrast enhanced digital mammography

AD Score and corresponding suggested BI-RADS category

$0-6$ benign: $0 \%$ malignant (BI-RADS 2)

$7-9$ probably benign: up to $2 \%$ (BI-RADS 3 )

10-14 low suspicious ( $>2$ to $\leq 10 \%$ ) (BI-RADS 4 A)

$15-17$ moderate suspicious ( $>10$ to $\leq 50 \%$ ) (BI-RADS $4 \mathrm{~B})$

$\geq 18$ highly suspicious $(>50 \%$ ) (BI-RADS $4 \mathrm{C}, 5)$

\#No odds ratio available as there are zero counts. Given the score of highest rank as $p<0.001$ in univariate analysis
Our study has a few limitations. Firstly, $10(10.6 \%)$ of 94 patients had only clinical diagnosis of benign lesions without histological diagnosis. As mentioned in the "Results" section, these 10 patients had additional evaluation with breast MRI for very subtle AD lesions on DBT. The remaining 4 (40\%) of 10 patients had no suspicious enhancement of AD on CEDM. The final BI-RADS was based on MRI assessment and DBT follow-up as there is no commercial DBT biopsy device in our early period of CEDM study. Fortuitously, all 10 patients were followed up for 2 years according to BI-RADS recommendation with no patient drop-out. These cases were shown to remain unchanged in $\mathrm{AD}$ size and morphology and were hence regarded as benign. Secondly, the sample size $(n=94)$ for this study may be limited by the availability of pure $\mathrm{AD}$ lesions at a single institution and the prediction model calculations were based on the Asian population at age of 30 69 years old which do not factor in clinical breast symptoms. This necessitates a larger study (i.e., multicenter study or systematic review) to corroborate our findings and to validate our prediction model in different populations and CEDM vendors. Thirdly, we did not include ultrasound imaging into the $\mathrm{AD}$ score. Breast ultrasound is well known for its high operator dependence, and 3D automated breast ultrasound seemed to have better performance in the detection of architectural distortion on the coronal plane [27]. Target ultrasound may help identify obvious mass lesions with spiculated margins and other non-calcified lesions on mammogram. However, the ADS and corresponding suggested BI-RADS lexicons can provide PPV prediction just based on mammographic features 

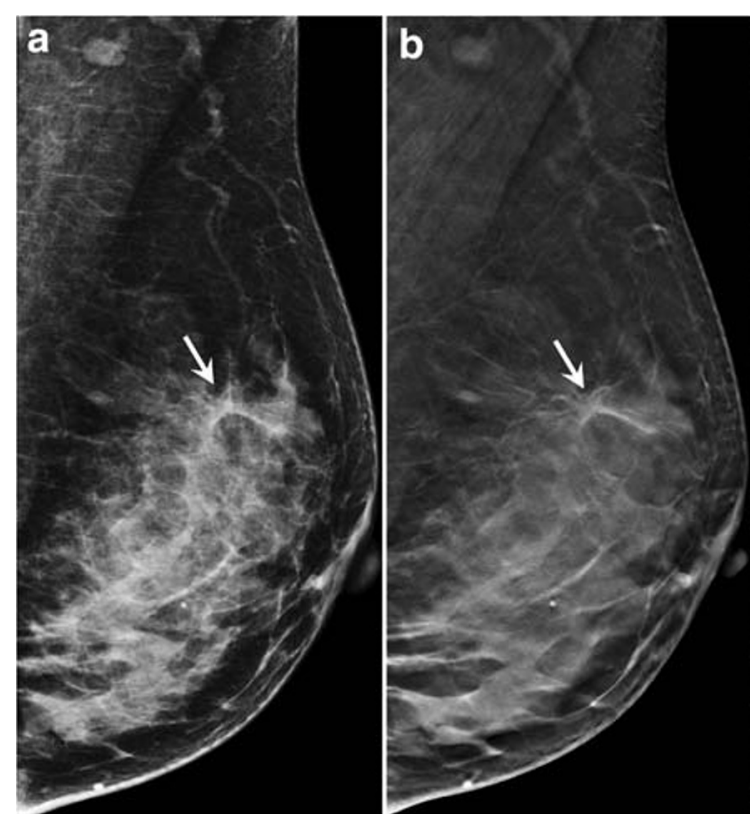

Fig. 5 A 49-year-old woman undergoing screening mammography. Mediolateral oblique (MLO) view of full-field digital mammography (FFDM) (a) and digital breast tomosynthesis (DBT) (b) depicting an architectural distortion (arrows) in the left breast. MLO view of contrast-enhanced digital mammogram (CEDM) (c) shows a 1.2-cm enhancing lesion (arrows) with the following characteristics: mild background parenchyma enhancement (score 1); size of lesion $>0.7 \mathrm{~cm}$ (score

alone and reduce bias between operators. In this study, surgical results of high-risk lesions such as atypical ductal hyperplasia (ADH) and lobular carcinoma in situ (LCIS) were classified as benign cases. The management of these high-risk lesions has been debated among experts; annual mammography or MRI screening is often considered necessary [28]. Lastly, the study did not analyze inter-reader agreement for the presented CEDM descriptive characteristics. In this risk prediction model study, the authors grouped as many subjective descriptive characteristics together to reduce potential bias and inter-reader disagreements (e.g., moderate and marked BPE are grouped together). The objective was to make as many variables as binary as possible to assist in better interpretation of CEDM images and adaptation of the risk prediction model. However, assessment of inter-agreement analysis of AD score as a whole could be assessed in a larger prospective study in the future.

\section{Conclusion}

The absence of suspicious enhancement of AD lesion at minimal or mild BPE indicates low risk of breast malignancy. For AD lesions which enhance, marked enhancement intensity and presence of enhancing spiculations are independent factors with highest associated risk of malignancy on multivariate

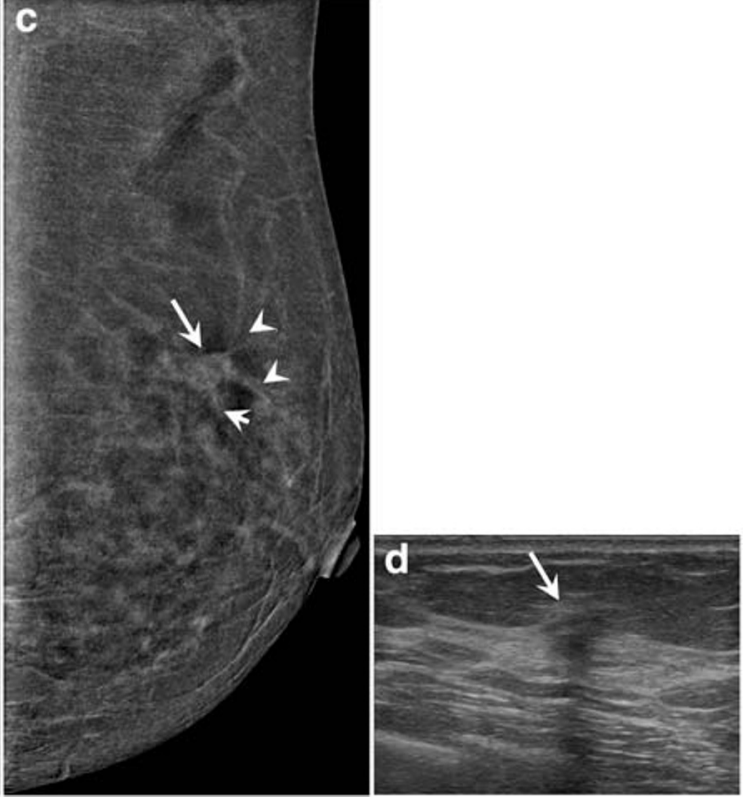

2); mass enhancement (score 7); moderate lesion enhancement (score 2); and presence of enhancing spiculations (score 4) (arrowheads). Architectural distortion score (ADS) is 16, and the lesion is categorized as BI-RADS 4B. (d) Correlative ultrasonographic image shows a hypoechoic mass (arrow) with posterior shadowing. Histopathology result: $0.8 \mathrm{~cm}$ invasive breast cancer

analysis. The ADS as mentioned can be a useful tool in assessment of BI-RADS categories of AD lesion at CEDM.

Acknowledgments Dr. Jennie Wong from the Division of Medical \& Scientific Communication, NUHS Research Support Unit, Singapore, for proofreading and editing the manuscript.

Dr. Chan Yiong Huak and Ms. Yap Qai Wen from the National University of Singapore (NUS) Medicine Biostatistics Unit for assistance in biostatistics.

Ms. Pei-Ying Lin from Kaohsiung Veterans General Hospital for preparing manuscript.

Funding The authors state that this work has not received any funding.

\section{Compliance with ethical standards}

Guarantor The scientific guarantor of this publication is Dr. Goh Yonggeng.

Conflict of interest The authors of this manuscript declare no relationships with any companies whose products or services may be related to the subject matter of the article.

Statistics and biometry Dr. Chan Yiong Huak and Ms. Yap Qai Wen from the National University of Singapore (NUS) Medicine Biostatistics Unit kindly provided statistical advice for this manuscript.

Informed consent Written informed consent was waived by the Institutional Review Board. 
Ethical approval Institutional Review Board approval was obtained.

\author{
Methodology \\ - retrospective \\ - cross-sectional study \\ - performed at one institution
}

Open Access This article is licensed under a Creative Commons Attribution 4.0 International License, which permits use, sharing, adaptation, distribution and reproduction in any medium or format, as long as you give appropriate credit to the original author(s) and the source, provide a link to the Creative Commons licence, and indicate if changes were made. The images or other third party material in this article are included in the article's Creative Commons licence, unless indicated otherwise in a credit line to the material. If material is not included in the article's Creative Commons licence and your intended use is not permitted by statutory regulation or exceeds the permitted use, you will need to obtain permission directly from the copyright holder. To view a copy of this licence, visit http://creativecommons.org/licenses/by/4.0/.

\section{References}

1. Knutzen AM, Gisvold JJ (1993) Likelihood of malignant disease for various categories of mammographically detected, nonpalpable breast lesions. Mayo Clin Proc 68(5):454-460

2. Venkatesan A, Chu P, Kerlikowske, Sickles EA, Smith-Bindman R (2009) Positive predictive value of specific mammographic findings according to reader and patient variables. Radiology 250(3): 648-657

3. Geller BM, Barlow WE, Ballard-Barbash R, et al (2002) Use of the American College of Radiology BI-RADS to report on the mammographic evaluation of women with signs and symptoms of breast disease. Radiology 222(2):536-542

4. Shaheen R, Schimmelpenninck CA, Stoddart L, Raymond H, Slanetz PJ (2011) Spectrum of diseases presenting as architectural distortion on mammography: multimodality radiologic imaging with pathologic correlation. Semin Ultrasound CT MR 32(4): 351-362

5. Bahl M, Baker JA, Kinsey EN, Ghate SV (2015) Architectural distortion on mammography: correlation with pathologic outcomes and predictors of malignancy. AJR Am J Roentgenol 205(6):13391345

6. Alshafeiy TI, Nguyen JV, Rochman CM et al (2018) Outcome of architectural distortion detected only at breast tomosynthesis versus 2D mammography. Radiology 288(1):38-46

7. Bahl M, Lamb LR, Lehman CD (2017) Pathologic outcomes of architectural distortion on digital 2D versus tomosynthesis mammography. AJR Am J Roentgenol 209(5):1162-1167

8. Dibble EH, Lourenco AP, Baird GL et al (2018) Comparison of digital mammography and digital breast tomosynthesis in the detection of architectural distortion. Eur Radiol 28(1):3-10

9. Buchberger W, DeKoekkoek-Doll P, Obrist P, Dünser M (1997) Value of MR tomography in inconclusive mammography findings. Radiologe 37(9):702-709

10. Gilles R, Guinebretiere JM, Lucidarme O et al (1994) Nonpalpable breast tumors: diagnosis with contrast-enhanced subtraction dynamic MR imaging. Radiology 191(3):625-631

11. Si L, Zhai R, Liu X, Yang K, Wang L, Jiang T (2016) MRI in the differential diagnosis of primary architectural distortion detected by mammography. Diagn Interv Radiol 22(2):141-150

12. Jochelson MS, Dershaw DD, Sung JS et al (2013) Bilateral contrast-enhanced dual-energy digital mammography: feasibility and comparison with conventional digital mammography and MR imaging in women with known breast carcinoma. Radiology 266(3):743-751

13. Li L, Roth R, Germaine P et al (2017) Contrast-enhanced spectral mammography (CESM) versus breast magnetic resonance imaging (MRI): a retrospective comparison in 66 breast lesions. Diagn Interv Imaging 98(2):113-123

14. Patel BK, Hilal T, Covington $\mathrm{M}$ et al (2018) Contrast-enhanced spectral mammography is comparable to MRI in the assessment of residual breast cancer following neoadjuvant systemic therapy. Ann Surg Oncol 25(5):1350-1356

15. Phillips J, Miller MM, Mehta TS et al (2017) Contrast-enhanced spectral mammography (CESM) versus MRI in the high-risk screening setting: patient preferences and attitudes. Clin Imaging 42:193-197

16. Suter MB, Pesapane F, Agazzi GM et al (2020) Diagnostic accuracy of contrast-enhanced spectral mammography for breast lesions: a systematic review and meta-analysis. Breast 53:8-17

17. Patel BK, Naylor ME, Kosiorek HE et al (2017) Clinical utility of contrast-enhanced spectral mammography as an adjunct for tomosynthesis-detected architectural distortion. Clin Imaging 46: 44-52

18. Durand MA, Wang S, Hooley RJ, Raghu M, Philpotts LE (2016) Tomosynthesis-detected architectural distortion: management algorithm with radiologic-pathologic correlation. Radiographics 36(2): 311-321

19. Pujara AC, Hui J, Wang LC (2019) Architectural distortion in the era of digital breast tomosynthesis: outcomes and implications for management. Clin Imaging 54:133-137

20. Vijapura C, Yang L, Xiong J, Fajardo LL (2018) Imaging features of nonmalignant and malignant architectural distortion detected by tomosynthesis. AJR Am J Roentgenol 211(6):1397-1404

21. Giess CS, Chikarmane SA, Sippo DA, Birdwell RL (2017) Clinical utility of breast MRI in the diagnosis of malignancy after inconclusive or equivocal mammographic diagnostic evaluation. AJR Am J Roentgenol 208(6):1378-1385

22. Macura KJ, Ouwerkerk R, Jacobs MA, Bluemke DA (2006) Patterns of enhancement on breast MR images: interpretation and imaging pitfalls. Radiographics 26(6):1719-1734 quiz 1719

23. Rudnicki W, Heinze S, Niemiec J et al (2019) Correlation between quantitative assessment of contrast enhancement in contrastenhanced spectral mammography (CESM) and histopathologypreliminary results. Eur Radiol 29(11):6220-6226

24. Nunes LW, Schnall MD, Orel SG (2001) Update of breast MR imaging architectural interpretation model. Radiology 219(2): 484-494

25. Hambly NM, Liberman L, Dershaw DD, Brennan S, Morris EA (2011) Background parenchymal enhancement on baseline screening breast MRI: impact on biopsy rate and short-interval follow-up. AJR Am J Roentgenol 196(1):218-224

26. Uematsu T, Kasami M, Watanabe J (2011) Does the degree of background enhancement in breast MRI affect the detection and staging of breast cancer? Eur Radiol 21(11):2261-2267

27. Vourtsis A, Kachulis A (2018) The performance of 3D ABUS versus HHUS in the visualisation and BI-RADS characterisation of breast lesions in a large cohort of 1,886 women. Eur Radiol 28(2):592-601

28. Lee CH, Dershaw DD, Kopans D et al (2010) Breast cancer screening with imaging: recommendations from the Society of Breast Imaging and the ACR on the use of mammography, breast MRI, breast ultrasound, and other technologies for the detection of clinically occult breast cancer. J Am Coll Radiol 7(1):18-27

Publisher's note Springer Nature remains neutral with regard to jurisdictional claims in published maps and institutional affiliations. 